\title{
The Therapeutic Relevance of Co-Targeting Wnt-Hh Signalling Pathways in Hepatocellular Carcinoma
}

\section{Anindita Tripathy ${ }^{1}$, Manoj Kumar Sahu ${ }^{2}$, Kanishka Uthansingh ${ }^{2}$, Ayaskanta Singh ${ }^{2}$, Jimmy Narayan ${ }^{2}$ and Ratna Kumari ${ }^{1 *}$}

${ }^{1}$ Disease Biology Lab, KIIT School of Biotechnology, KIIT University, Bhubaneswar, India

${ }^{2}$ Department of Gastroenterology and Hepatobiliary Sciences, IMS and SUM Hospital, Bhubaneswar, India

*Corresponding Author: Ratna Kumari, Disease Biology Lab, KIIT School of

Biotechnology, KIIT University, Bhubaneswar, India.

E-mail: ratnaintouch@gmail.com

DOI: $10.31080 /$ ASGIS.2020.03.0125

\section{Received: January 02, 2020}

Published: February 27, 2020

(C) All rights are reserved by Ratna Kumari., et al.

\section{Abstract}

Hepatocellular carcinoma (HCC) is one of the most commonly diagnosed primary liver cancer and is refractory to most of the chemotherapeutic drugs available. Multiple signalling pathways are deregulated in HCC, and two important developmental signalling pathways which are deregulated in HCC are Wnt and Hh signalling pathways. In various cancer models both pathways have been found to interact with each other at different levels and this interaction is known to play important role in the pathophysiology of those cancers like pancreatic cancer, colon cancer, and basal cell carcinoma. Previously we have shown that the Wnt and Hh pathways correlate positively and play an important role in the progression of hepatocarcinogenesis in rodent model. Our previously published data suggested that the investigation of cross-talk between these two pathways holds potential for identification of better HCC treatment strategies. In the present study we utilized pharmacological inhibitors of Wnt and Hh signalling pathways to unravel the functional interaction between these two pathways. We studied the impact of inhibition or up-regulation of Wnt signalling pathway on Hh signalling pathway outcome. We also investigated the chemo-preventive action of Sulindac Sulphide, a Wnt/ $\beta$-catenin pathway inhibitor on tumorigenesis in animal model of hepatocarcinogenesis. Our study elaborated the nature of cross-talk between these two well known aberrant signalling pathways in HCC which would further pave the way for development of better combination therapy approach for targeting these two pathways in HCC.

Keywords: Hepatocellular Carcinoma; Sulindac Sulphide; KAAD-Cyclopamine; Wnt/ $\beta$-catenin; Hh/Gli1

\section{Introduction}

Hepatocellular carcinoma (HCC) is one of the most commonly diagnosed primary liver cancer and is also a major cause of cancer-related mortality across the world [1]. Additionally, it is one of the cancers which is admittedly refractory to chemotherapy [2] to radiotherapy and to combination treatment [3]. The aetiology of HCC is diverse including viral factors like infection with hepatitis B or hepatitis $\mathrm{C}$ virus infection (HBV and $\mathrm{HCV}$ ); non-viral factors like alcohol consumption, obesity and other metabolic syndrome; aflatoxin exposure; hereditary factor, and genetic predisposition like mutations in important signalling pathway genes [1,4]. Hepatocarcinogenesis is a multistep process, progressing from a normal hepatocyte to transformed phenotype as a result of the accumulation of aberrant genetic and epigenetic modifications and activation of various signalling pathways [5].

Two major signalling pathways found to be deregulated in HCC are Wnt and Hedgehog (Hh) pathways [6]. These two pathways play key roles in embryogenesis, morphogenesis, tissue patterning, angiogenesis, stem cell maintenance, tumorigenesis, and drug resistance [7]. In various cancer models both pathways have been found to interact with each other at different levels and this interaction is known to play important role in the pathophysiology of those cancers like Pancreatic cancer [8], colon cancer [9] and in basal cell carcinoma [10]. Our previously published work demonstrated the role of Wnt and Hh pathway in progression of hepatocarcinogenesis in rodent model [6]. Previously we have shown the stepwise comprehensive changes in the molecular expression pattern of Wnt-Hh signalling pathways at different stages of HCC viz. Initiation, Promotion, and Progression. Our study provided evidence that activation of $\mathrm{Wnt} / \beta$-catenin pathway is the key step in the process of hepatocarcinogenesis; where $\beta$-catenin is the transcriptional co-activator which plays important role in the initiation of hepatocarcinogenesis. We also observed activation of Hh signalling pathway in rodent hepatocarcinogenesis model. The activation of these two pathways in HCC was also confirmed by other investigators [11]. Indeed, these two pathways are known to interact with each other at different levels in various cancers, but the level and nature of the interaction between these pathways is not completely known in HCC. 
Various drugs have been developed to target Wnt and Hh signalling pathways separately in different cancers including HCC, but most of these drugs could not reach clinical trials; few which have reached clinical trials have shown reduced efficacy and compromised safety profile $[12,13]$. Development of resistance to a particular drug during the trial period is also an additional drawback as observed in case of Basal Cell Carcinoma [10]. Given the fact it is always necessary to investigate other potential druggable targets and better effective inhibitors of already known aberrant signalling pathways in HCC.

Sulindac sulphide (SS) is NSAID and well known for its chemopreventive effect on colorectal cancer [14]. The specific mechanism of Wnt inhibition by SS is not known, but the chemical is known to inhibit COX enzyme which is required for Prostaglandin E2 production and PGE2 is in-turn responsible for decreased $\beta$-catenin degradation. The decrease in COX enzyme by SS leads to the subsequent decrease in the prostaglandin E2 consequently enhancing $\beta$-catenin degradation [15]. However, the precise mechanism of SS for the chemopreventive action is unknown.

Similarly, KAAD-Cyclopamine is small molecule inhibitor of $\mathrm{Hh}$ pathway, and it is known to suppress Smo receptor [16]. Aberrant Hh signalling pathway activation is reported in a number of malignancies [17] including HCC $[6,13]$. Gain-of-function mutation of Smo receptor is one of the mechanisms of ligand independent $\mathrm{Hh}$ activation [18]. So suppression of Smo receptor and consequent Hh pathway is one of the strategies for targeted cancer treatment Various Smo inhibitors are developed and have been used for cancer treatment in different clinical trials with an acceptable efficacy and safety. But here also the development of resistance to inhibitor is evident [19]. All these facts infer towards the development of better inhibitors of these commonly aberrant pathways in cancer. Furthermore, combination of inhibitors which can simultaneously target two pathways for a better therapeutic efficacy should be explored.

GSK3 $\beta$ is the common intermediate between these two pathways and it is known to be inhibit Wnt and Hh signalling pathways $[6,9]$. $\mathrm{LiCl}$ is a potent well known inhibitor of Gsk3 $\beta[20,21]$. By this way $\mathrm{LiCl}$ is implicated to activate Wnt and Hh signalling pathway and promote tumorigenesis. $\mathrm{LiCl}$ is known to promote multiple myeloma; however, the exact mechanism is still ambiguous [22]. $\mathrm{LiCl}$ is a well-known drug for the treatment of bipolar disease, depression, and specifically for mania [23]. However, the role of $\mathrm{LiCl}$ on Wnt and Hh pathway in context of HCC has not been investigated.

In the present study we identified the cross-talk between the two pathways in a human hepatocellular carcinoma cell-line Hep3B. Investigating Wnt and Hh interaction would give valuable information regarding the better treatment strategies for HCC. We also utilized DEN induced wistar rat model of hepatocarcinogen- esis to unravel the chemo preventive impact of Wnt pathway inhibition and its impact on $\mathrm{Hh}$

\section{Material and Methods}

Cell culture, treatment and transient transfection

Human HCC cell line Hep3B was obtained from National Centre for Cell Science, Pune, India. Cells were plated in 6 well plates and cultured in DMEM (GIBCO, Invitrogen) media, supplemented with penicillin $(100 \mathrm{U} / \mathrm{ml})$, streptomycin $(100 \mathrm{U} / \mathrm{ml})$ and $10 \%$ fetal bovine serum at $37^{\circ} \mathrm{C}$ in $5 \% \mathrm{CO}_{2-} 95 \%$ air atmosphere in a humidified incubator. Cells were subcultured in every 5 to 6 days as per experimental requirements. Hep3B cells were treated with $10 \mathrm{mM}$ of Lithium Chloride (LiCl, Himedia), 2-2.5uM of KAAD cyclopamine (KC, Sigma Aldrich) and 75-100uM of Sulindac sulphide (SS, Sigma Aldrich) and harvested at specified times. For siRNA inhibition studies, the cells were transfected with validated human $\beta$-catenin (CTNNB1) siRNA or negative control siRNA (Ambion) at a final concentration of $100 \mathrm{nM}$ in the presence of an Oligofectamine reagent as per the manufacturer's instruction. After transfection, cells were treated with inhibitors and harvested at 48hrs for protein and mRNA extraction and additional analysis. All experiments were performed in triplicates and representative results are reported.

\section{Cell viability assay}

Cell viability assay was performed to detect the viable proliferating cells. All experiments were performed in triplicate Briefly, 8000 cells were seeded in 96 well plates and treated with Licl, KC and SS at various concentrations for 24, 48 and 72hrs. Cells were also transfected with $\beta$-catenin siRNA. After treatment and transfection MTT reagent was added and incubated for 4 hrs in dark for formazan crystal formation. Then the crystals were dissolved and the absorbance was measured at $570 \mathrm{~nm}$ in a microplate reader. The Mean \pm SEM absorbance units obtained from three experiments for each group were compared for statistical significance. The data were normalized to their respective controls and are presented as a bar graph.

\section{Colony formation assay}

For anchorage-dependent colony formation assay, 600 cells were seeded in 6well plates and incubated for $24 \mathrm{hr}$ and treated with inhibitors till colonies were formed (10-12days). The culture media was replaced every 2-3 days. The colonies were fixed and stained with crystal violet for $1 \mathrm{hr}$ at room temperature. The wells were washed with distilled water, dried overnight at room temperature and the images were taken. Finally the stain was dissolved in $10 \%$ glacial acetic acid and the absorbance of the released dye was measured at $590 \mathrm{~nm}$.

\section{Protein extraction and western blot analysis}

Protein extraction and western blot analysis was performed as per laboratory protocol. Briefly, Hep3B cells after $\mathrm{LiCl}, \mathrm{KC}$, SS and siRNA treatment were used for total cell lysate preparation by using the conventional homogenization method by RIPA Lysis buffer (50 mM Tris-Cl pH 7.4, 150 Mm Nacl, 1\% NP-40, 0.25\% sodium 
desoxycholate, 1 mM EDTA, $1 \mathrm{mM}$ PMSF, $1 \mathrm{mM}$ Na3V03,1mM EGTA,1x PIC, MQ). The concentration of protein was determined by Bradford Protein Assay, with Bovine serum albumin as standard. Aliquotes of samples were stored at $-80^{\circ} \mathrm{C}$ until use. 50 or 70 $\mu \mathrm{g}$ of protein was resolved by $10 \%$ SDS-Polyacrylamide Gel Electrophoresis analysis using the mini-PROTEIN 3-electrophoresis module assembly (Bio-Rad) and transferred to immobilon PVDF membranes. The membrane was blocked at $5 \%$ skimmed milk in TBST for $2 \mathrm{hrs}$ at RT and then probed with the primary antibodies (1:1000or 1:2000 as required) for $3 \mathrm{hrs}$ at RT or at $4^{\circ} \mathrm{C}$ overnight. The membrane was washed with TBST and probed with HRPconjugated secondary antibody $(1: 2,500)$ for $2 \mathrm{hrs}$ at RT. The blots were visualized by enhance chemiluminescence using X-ray film. Densitometric analysis on blots was performed by Image-J software and the average integrated optical density in treated group was normalized to loading control $\beta$-actin at the corresponding times.

\section{Immuno cytochemistry}

Hep3B cells were grown on glass coverslips to $60 \%-80 \%$ confluency in 24 or 12 well plates and treated with $\mathrm{LiCl}(10 \mathrm{mM}), \mathrm{KC}$ (2.5 uM), SS (100 uM) and transfected with $\beta$-catenin siRNA for $48 \mathrm{hrs}$. The media was decanted and the cells grown on the coverslips were washed with $1 \mathrm{X}$ PBS for three times. Cells were then fixed with 3.7\% paraformaldehyde for 5-7 minutes at RT followed by $1 \mathrm{X}$ PBS wash. Cells were made permeable with $0.1 \%$ TritonX-100 for 10 minutes at RT and blocked with $1 \%$ Bovine Serum Albumin for 30 minutes. Then cells were probed with primary antibody $(1: 1000$ or $1: 2000)$ at $4{ }^{\circ} \mathrm{C}$ overnight. This was followed by washing with $1 \mathrm{X}$ PBS and probing with FITC fluorophore tagged secondary antibodies (1:3000) for $1 \mathrm{hr}$ at RT. Cells were counterstained with 4,6-diamidino-2phenylindole (DAPI), washed, mounted and viewed under fluorescence microscope Olympus (BX 61) and capturing of the images were done using Image Pro Express software. The cell fluorescence was measured using ImageJ software. Corrected Total Cell Fluorescence (CTCF) was calculated using the formula.

CTCF $=$ Integrated Density $-($ Area of selected cell X Mean fluorescence of background readings) ("Measuring cell fluorescence using ImageJ — The Open Lab Book v1.0”, n.d.).

\section{In-vivo experiments and hepatocarcinogenesis model}

In vivo studies were performed with male Albino Wistar rats of 120-150g body weight, which were procured from the laboratory animal facility of KIIT School of Biotechnology. They were housed in standard temperature/humidity conditions and environment (12hr light/dark cycle). All animals were provided standard pellet diet and water ad libitum. All the experimental protocols were approved by the Institutional Animal Ethics Committee (IAEC, KIIT School of Biotechnology, Bhubaneswar, India).

The rats were randomly and evenly allocated into three groups, six rats in each group. Groups were assigned as Control, DEN+CCl4 group and SS treated group animals. All animals were acclimatized for two weeks before starting the experiment. The DEN (Cat: N0258, Sigma Aldrich) + CCl4 model of rat hepatocarcinogenesis was followed in our experiment. N-Nitrosodiethylamine (DEN) was administered in DEN+CCl4 group and SS group as $100 \mathrm{mg} / \mathrm{kg}$ body weight per week for three consecutive weeks. After 1 week of recovery period, the promoting reagent CCL4 (Carbon tetrachloride) was given $2 \mathrm{ml} / \mathrm{kg}$ body weight of rats weekly twice for consecutive 8 weeks. For SS treated group, Sulindac Sulphide (SS) $12 \mathrm{mg} /$ $\mathrm{kg}$ body weight per day was administered intraperitoneally for 10 days before CCL4 injection. At the completion of the 8 week treatments, and after latency period of two weeks we observed altered hepatic foci or morphologically recognizable lesions in DEN treated animals. The rats were sacrificed under anesthesia and before sacrificed the total food-water intake and body weights were recorded.

Reverse transcription quantitative polymerase chain reaction (RT-qPCR) analysis

Total RNA was extracted from Hep3B cells, rat liver tissues and HCC patients FNAC samples (Control-4 and HCC patient-23)with TRIzol reagent in accordance with the manufacturer's instructions. Reverse transcription was performed in total volume of $20 \mu \mathrm{l}$ using $2 \mu \mathrm{g}$ of total RNA by Verso c-DNA synthesis kit. To quantify the changes in m-RNA level reverse transcription PCR was performed on the CFX ConnectTM Real-Time PCR Detection System (Life-Technology, BIORAD) by using GoTaq ${ }^{\circledR}$ Green Master Mix. PCR primers are designed based on human mRNA sequence. PCR products were separated by electrophoresis in $1 \%$ agarose gel, visualized by $0.5 \mathrm{ug} / \mathrm{ml}$ ethidium bromide staining for $40 \mathrm{mnts}$. The gel image and intensity of each band was measured by GEL-DOC Image software (BIO-RAD). q-PCR was performed by using Sso Fast EvaGreen Supermix (BIORAD) with the following cycling conditions: $95^{\circ} \mathrm{C}$ for $5 \mathrm{~min}$, followed by 32 cycles of $95^{\circ} \mathrm{C}$ for $15 \mathrm{sec}$ and $60^{\circ} \mathrm{C}$ for $25 \mathrm{sec}$. Glyceraldehyde 3-phosphate dehydrogenase (GAPDH) gene was used as the internal control. The primer details are described in table 1.

\section{Elisa}

The detection of whole cell lysates and rat tissue lysates was done by Indirect ELISA method. Briefly, the protein antigen $(1 \mu \mathrm{g} /$ $\mathrm{ml}$ conc.) was mixed with coating buffer $(0.05 \mathrm{M})$ and coated onto 96 well microplate.

The plate was then incubated overnight at $4^{\circ} \mathrm{C}$ followed by washing with $1 \mathrm{X}$ PBST and then blocking with $1 \%$ BSA at room temperature for $2 \mathrm{hrs}$. Then primary antibodies (1:2000 dilution) were added into each well and incubated $2 \mathrm{hrs}$ at room temperature followed by washing thrice with $1 \mathrm{X}$ PBST. The wells were then incubated with secondary HRP linked antibody (1:2500 and 1:5000 dilutions according to the primary antibody) for 45 mints at room temperature. After washing thrice with 1X PBST, 2,2'-azino-bis (3-ethylbenzothiazoline-6-sulphonic acid) or ABTS substrate solution was added and absorbance was read at $410 \mathrm{~nm}$ using ELISA microtitreplate reader (EPOCH). 


\begin{tabular}{|l|c|c|c|c|}
\hline \multicolumn{1}{|c|}{ Genes } & Sense & Anti-Sense & Annealing Temp. & No. of cycles \\
\hline $\boldsymbol{\beta}$-catenin & AACGGCTTTCGGTTGAGCTG & TGGCGATATCCAAGGGCTTC & $60^{\circ} \mathrm{C}$ & 30 \\
\hline cMyc & CCAGGACTGTATGTGGAGCG & CCTGAGGACCAGTGGGCTGT & $56.6^{\circ} \mathrm{C}$ & 34 \\
\hline Wnt3 & GGAGAAACGGAAGGAGAAATG & GAGAGACGTTAGTTGAGAAAGAAGC & $58^{\circ} \mathrm{C}$ & 32 \\
\hline Gli1 & GGGATGATCCCACATCCTCAGTC & CTGGAGCAGCCCCCCCAGT & $56.6^{\circ} \mathrm{C}$ & 34 \\
\hline Shh & GATGTCTGCTGCTAGTCCTCG & CACCTCTGAGTCATCAGCCTG & $55.3^{\circ} \mathrm{C}$ & 34 \\
\hline SmoH & GTTCTCCATCAAGAGCAACCAC & CGATTCTTGATCTCACAGTCAGG & $55.3^{\circ} \mathrm{C}$ & 34 \\
\hline Ptch1 & CAGAGAAGGCTTGTGGCCAC & GCTCAATGACTTCCACCTTCG & $56.2^{\circ} \mathrm{C}$ & 34 \\
\hline LRP5 & ACCGGAACCACGTCACAG & GGGTGGATAGGGGTCTGAGT & $54.3^{\circ} \mathrm{C}$ & 32 \\
\hline LRP6 & AGGCACTTACTTCCCTGCAA & GGGCACAGGTTCTGAATCAT & $54.2^{\circ} \mathrm{C}$ & 32 \\
\hline Fzd1 & GTGAGCCGACCAAGGTGTAT & CAGCCGGACAAGAAGATGAT & $53.6^{\circ} \mathrm{C}$ & 30 \\
\hline GAPDH & ATCTTCCAGGAGCGAGATCCC & CGTTCGGCTCAGGGATGACCT & $58^{\circ} \mathrm{C}$ & 30 \\
\hline
\end{tabular}

Table 1

Statistical analysis

The data presented is the Mean \pm SEM of three independent experiments. Changes in gene expressions and the statistical analysis for ELISA readouts were analyzed by two way analysis of variance (ANOVA) using Grpah-Pad Prism5. The fold change of m-RNA was used as variables to compare samples between different treatment groups. ${ }^{*} \mathrm{p}<0.05,{ }^{* *} \mathrm{p}<0.001,{ }^{* * *} \mathrm{p}<0.0001$ were considered to be significant.

\section{Results}

Inhibition of Wnt pathway in Hep3B cells by using sulindac sulphide (SS)

In order to check the effect of SS on Wnt pathway in Hep3B cells, we treated the cells with different dosage of SS for 24, 48 and $72 \mathrm{hr}$ post-treatment growths. Our results demonstrated significant reduction in cell viability for all dosage at 48 and $72 \mathrm{hr}$ (Figure 1A). The long-term cell viability like colony formation assay also demonstrated the similar results (Figure 1B). We also performed immunocytochemistry and demonstrated that the expression level of different molecules of Wnt signaling pathway ( $\beta$-catenin, Wnt3, c-Myc, Fzd, LRP-5/6) got reduced after SS treatment (Figure 1C). These results were complemented with other techniques for the mRNA and protein level expression analysis of Wnt pathway molecules (Figure 1D-G).

Inhibition of Hh pathway in Hep3B cells by using KAAD-cyclopamine (KC)

After checking the effect of SS treatment on Hep3B cells we next demonstrated the impact of inhibition of Hh signaling pathway in Hep3B cells. The cells were treated simultaneously with different dosage of KC and allowed for 24, 48, and 72hrs post-treatment growths. Our results demonstrated significant reduction in cell viability for all dosage at 48 and $72 \mathrm{hr}$ (Figure 2A). The long-term cell viability like colony formation assay also demonstrated the similar results (Figure 2B). We also performed immunocytochemistry and demonstrated that the expression level of different molecules of Hh signaling pathway (Gli1, Ptch1, SHH, SmoH) got reduced after $\mathrm{KC}$ treatment (Figure 2C). These results were complemented with

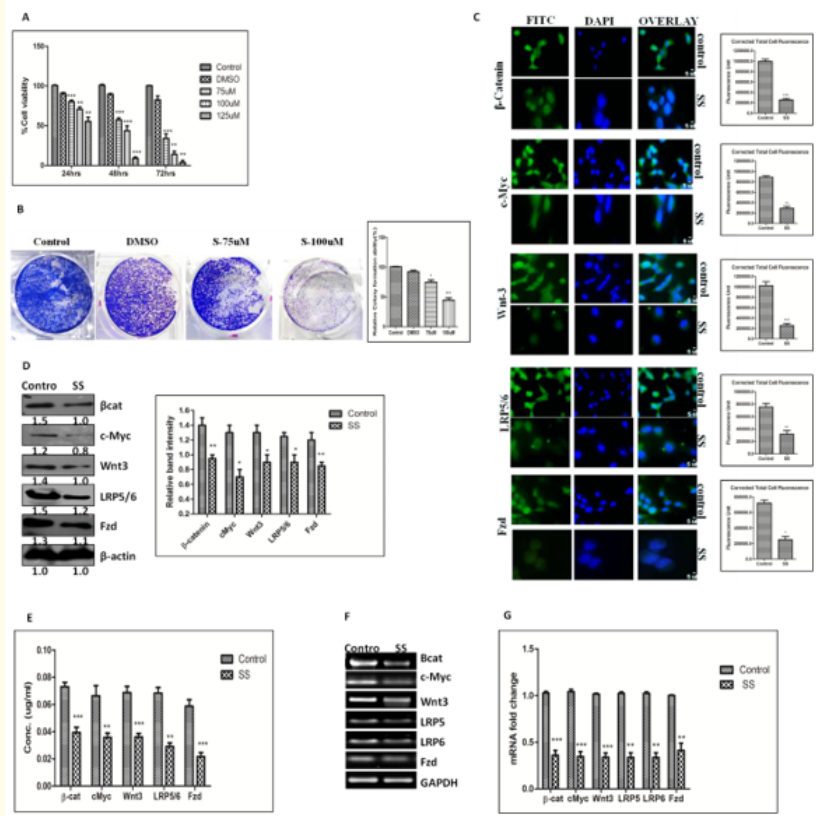

Figure 1: Inhibition of Wnt pathway in Hep3B cells by using Sulindac sulphide (SS). (A) Cell proliferation assaay of Hep3B cells after SS treatment. After 24, 48 and $72 \mathrm{hr}$, the MTT assay was carried out as described in materials and methods. The cell viability is directly proportional to the amount of formazan crystal formation. (B) Effect of SS on colony formation of Hep3B cells. The relative colony formation ability (\%) was measured and shown in the graph. (C) ICC staining of Wnt pathway molecules in Hep3B cells after SS treatment. Corrected Total Cell Fluorescence showing the quantification of Wnt molecules. (D) Western blot analysis in whole cell lysates and the graph showing relative band intensity. (E) Analysis of Wnt molecules in cell lysates by ELISA. (F-G) The relative mRNA expression with respect of GAPDH for Wnt pathway molecules in Hep3B cells after SS treatment. Data represented are representative of three independent experiments performed in triplicates and expressed as Mean \pm SE, ${ }^{*} \mathrm{p}<0.05,{ }^{* *} \mathrm{p}<0.001,{ }^{* * *} \mathrm{p}<0.0001$.

other techniques for the mRNA and protein level expression analysis of Hh pathway molecules (Figure 2D-G). 


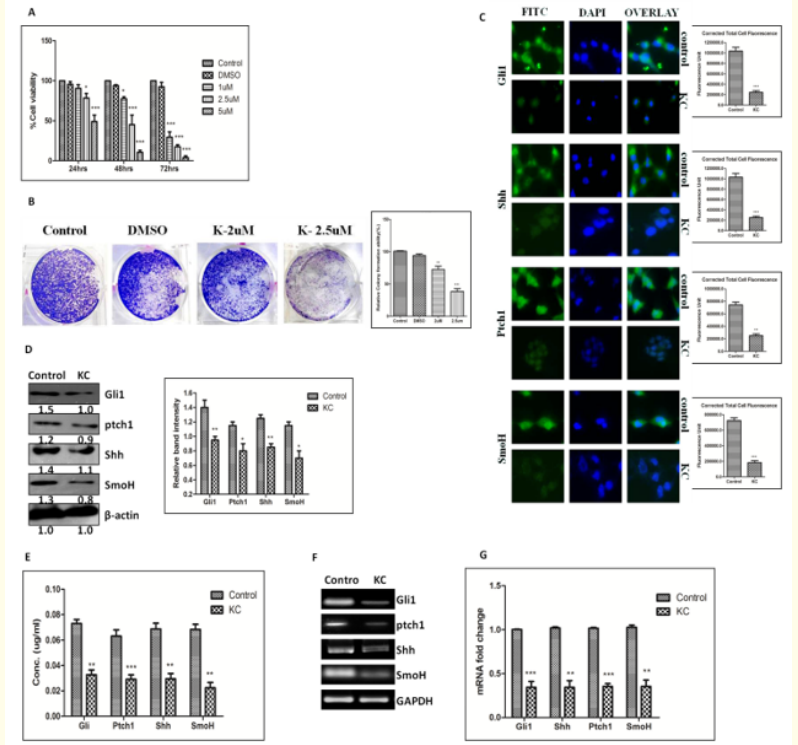

Figure 2: Inhibition of Hh pathway in Hep3B cells by using KAADCyclopamine (KC). (A) Cell proliferation assaay of Hep3B cells after $\mathrm{KC}$ treatment for 24,48 and $72 \mathrm{hr}$. The cell viability is directly proportional to the amount of formazan crystal formation. (B) Effect of KC on colony formation of Hep3B cells. The relative colony formation ability (\%) was measured and shown in the graph. (C) ICC staining of Hh pathway molecules in Hep3B cells after KC treatment. Corrected Total Cell Fluorescence showing the quantification of Hh molecules. (D) Western blot analysis in whole cell lysates and the graph showing relative band intensity. (E) Expression analysis of Hh molecules by ELISA. (F-G) The decreasing relative mRNA expression with respect of GAPDH for Hh pathway molecules in Hep3B cells after KC treatment. Data represented are representative of three independent experiments performed in triplicates and

expressed as Mean $\pm \mathrm{SE},{ }^{*} \mathrm{p}<0.05,{ }^{* *} \mathrm{p}<0.001,{ }^{* * *} \mathrm{p}<0.0001$.

Inhibition of Wnt-Hh pathway in Hep3B cells by using SS+KC

Given the significant reduction in the expression level of Wnt and Hh signaling pathway molecules after treatment with their respective inhibitors, we next investigated if there is any synergism between these two inhibitors via. cell viability assay. Both shortterm and long-term assays revealed the synergistic effect on cell viability (Figure 3A-B). The expression level of different Wnt-Hh pathway molecules were also reduced after combined treatment with their inhibitors (Figure 3C). The expression of different molecules was also checked at mRNA and protein level after combined treatment with the inhibitors (Figure 3D-G).

Activation of Wnt pathway by using LiCl simultaneously enhanced Hh pathway in Hep3B cells

Next we checked the interaction between Wnt-Hh pathways by exogenously activating Wnt pathway by $\mathrm{LiCl}$. $\mathrm{LiCl}$ is known to activate Wnt pathway via. inhibition of GSK3 $\beta$. We first performed MTT assay and screened different $\mathrm{LiCl}$ concentrations for its effect on Wnt activation in Hep3B cells. Our results demonstrated that

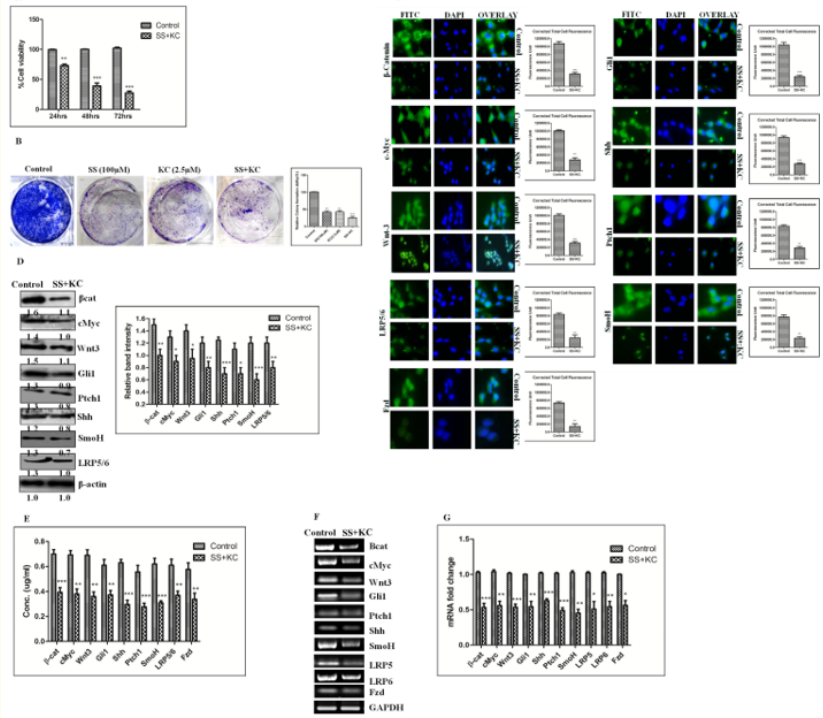

Figure 3: Inhibition of Wnt-Hh pathway in Hep3B cells by using SS+KC. (A) Cell proliferation assaay of Hep3B cells after SS+KC treatment for 24,48 and $72 \mathrm{hr}$. The cell viability is directly proportional to the amount of formazan crystal formation. (B) Effect of SS+KC on colony formation of Hep3B cells. The relative colony formation ability (\%) was measured and shown in the graph. (C) ICC staining of Wnt and Hh pathway molecules in Hep3B cells after SS+KC treatment. Corrected Total Cell Fluorescence showing the quantification of Wnt-Hh molecules. (D) Western blot analysis of Wnt-Hh pathway molecules and the graph showing relative band intensity. (E) Expression analysis of Wnt-Hh molecules by ELISA. (F-G) The relative mRNA expression with respect of GAPDH for Wnt-Hh pathway molecules in Hep3B cells after SS+KC treatment. Data represented are representative of three independent experiments performed in triplicates and expressed as Mean $\pm \mathrm{SE}$, ${ }^{*} \mathrm{p}<0.05,{ }^{* *} \mathrm{p}<0.001,{ }^{* * *} \mathrm{p}<0.0001$.

after treatment with $10 \mathrm{mM} \mathrm{LiCl}$ for $48 \mathrm{hr}$ the Wnt pathway got significantly up-regulated (Figure 4A). Our results also revealed visual increase in the expression level of $\beta$-catenin and other molecules of Wnt pathway (Figure 4B) and this increase was simultaneously associated with the increased expression of Hh pathway molecules like Gli1 and Ptch1 (Figure 4B). We also checked the mRNA and protein level expression of different molecules of Wnt and Hh pathways. Figure 4C-D represents molecular expression changes at protein level and figure $4 \mathrm{E}-\mathrm{F}$ represents molecular expression changes at mRNA level for both Wnt and Hh pathways.

LiCL treatment partially reversed $\beta$-cat siRNA mediated suppression of Wnt pathway in Hep3B cells

We next investigated the impact of LiCl after siRNA mediated suppression of $\beta$-catenin and consequent Wnt pathway. Interestingly, our results demonstrated the partial reversal of siRNA mediated suppression of $\beta$-catenin. The results revealed that the inhibition of $\beta$-catenin after siRNA treatment was enhanced when the 


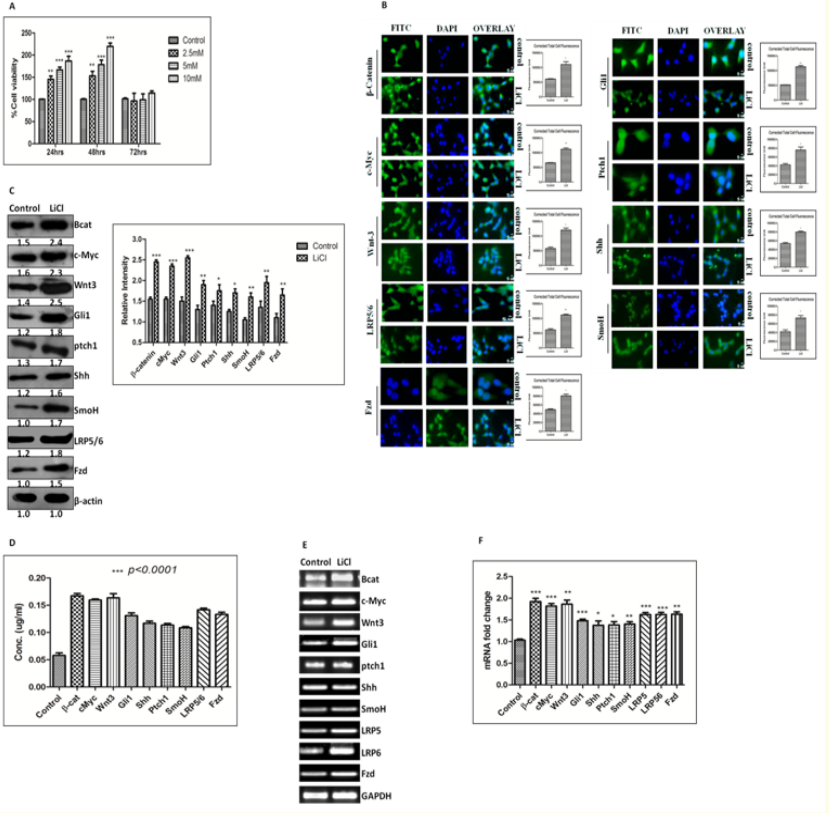

Figure 4: Activation of Wnt pathway by using LiCl simultaneously enhanced Hh pathway in Hep3B cells. (A) Cell proliferation assaay of Hep3B cells after LiCl treatment for 24, 48 and $72 \mathrm{hr}$. The cell viability is directly proportional to the amount of formazan crystal formation. (B) ICC staining of Wnt and Hh pathway molecules in Hep3B cells after LiCl treatment. Corrected Total Cell Fluorescence showing the quantification of Wnt-Hh molecules. (C) Western blot analysis of Wnt-Hh pathway molecules after LiCl treatment and the graph showing relative band intensity. (D) Expression analysis of Wnt-Hh molecules by ELISA. (E-F) The relative increasing mRNA expression with respect of GAPDH for Wnt-Hh pathway molecules in Hep3B cells after LiCl treatment. Data represented are representative of three independent experiments performed in triplicates and expressed as Mean $\pm \mathrm{SE},{ }^{*} \mathrm{p}<0.05,{ }^{* *} \mathrm{p}<0.001,{ }^{* * *} \mathrm{p}<0.0001$.

cells were also treated with SS or KC. Furthermore, this enhanced suppression of $\beta$-catenin was partially reversed when we treated the cells with $\mathrm{LiCl}$ as visualized after ICC staining of $\beta$-catenin (Figure $5 \mathrm{~B}$ ). The result was also complemented with other techniques like Western blot (Figure 5C), ELISA (Figure 5D), RT-PCR (Figure 5E) and qRT-PCR (Figure 5F) of cell-lysates. We also checked the expression level of direct transcriptional targets of Hh pathway like Gli1 and Ptch1. Notably, the expression of these Hh pathway molecules also changed simultaneously as that of $\beta$-catenin expression after siRNA and inhibitor treatments. Figure $6 \mathrm{~A}$ represents ICC staining of Gli1, Ptch1, and SHH after indicated treatments and figure 6B-E represents the same result complemented with other techniques.

Enhaced Wnt/ $\beta$-catenin activation correlates with increased CRD-BP and Gli1 in HCC patient's biospecimen

CRD-BP is an oncofetal protein and it has been found to be overexpressed in a number of cancers including HCC [24]. We observed increased level of Wnt/ $\beta$-catenin pathway was simultaneously as-

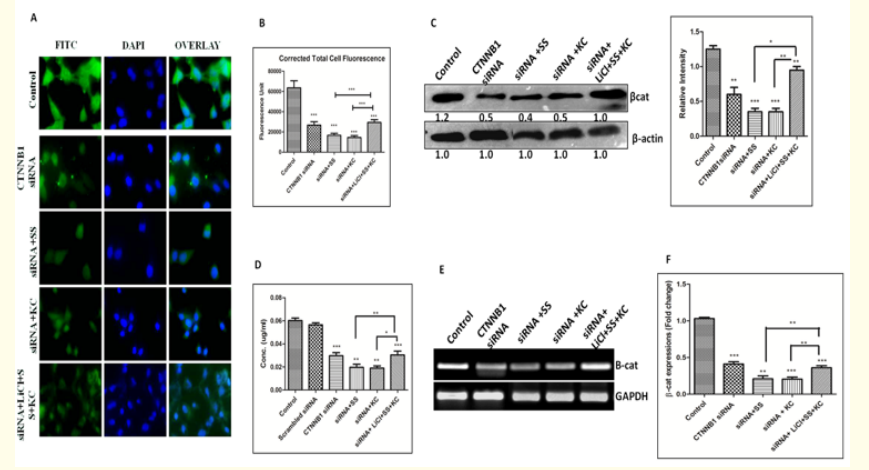

Figure 5: Licl treatment partially reversed $\beta$-cat siRNA mediated suppression of Wnt pathway in Hep3B cells. (A-B) ICC staining of $\beta$-catenin after $48 \mathrm{hr}$ of SS, KC and LiCl treatment in CTNNB1 transfected Hep3B cells. Corrected Total Cell Fluorescence showing the quantification of $\beta$-cat. (C) Western blot analysis of $\beta$-cat after treatment and the graph showing relative band intensity. (D) Expression analysis of $\beta$-cat by ELISA. (E-F) The relative mRNA expression with respect of GAPDH for $\beta$-cat in CTNNB1 transfected Hep3B cells. Data represented are representative of three independent experiments performed in triplicates and expressed as Mean $\pm \mathrm{SE},{ }^{*} \mathrm{p}<0.05,{ }^{* *} \mathrm{p}<0.001,{ }^{* * *} \mathrm{p}<0.0001$.
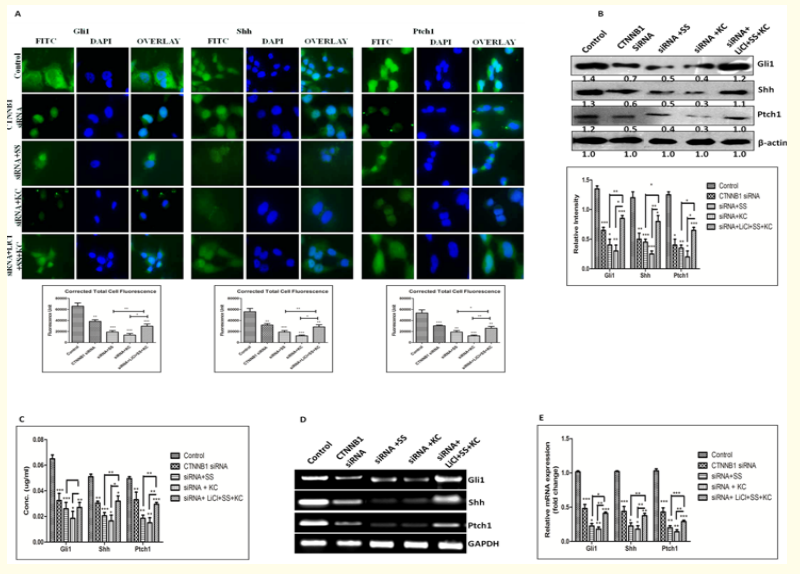

Figure 6: Wnt inhibition resulted into reduced Hh signaling in Hep3B cells. (A) ICC staining of Gli1, Shh and Ptch1 after 48hr of SS, $\mathrm{KC}$ and LiCl treatment in CTNNB1 transfected Hep3B cells. Corrected Total Cell Fluorescence showing the quantification of $\beta$-cat. (B) Western blot analysis of these molecules after treatment and the graph showing relative band intensity. (C) Expression analysis of Gli1, Shh and Ptch1 by ELISA. (D-E) The relative mRNA expression with respect of GAPDH for Gli1, Shh and Ptch1 in CTNNB1 transfected Hep3B cells. Data represented are representative of three independent experiments performed in triplicates and expressed as Mean $\pm \mathrm{SE},{ }^{*} \mathrm{p}<0.05,{ }^{* *} \mathrm{p}<0.001,{ }^{* * *} \mathrm{p}<0.0001$.

sociated with enhanced expression of CRD-BP in Hep3B cells after $\mathrm{LiCl}$ treatment (Figure 7A-B). We elaborated this finding in FNAC samples of HCC patients' and we observed increased $\beta$-catenin in these patients was also associated with increased expression of CRD-BP and Gli1 as compared to control FNAC samples (Figure 7CD). 


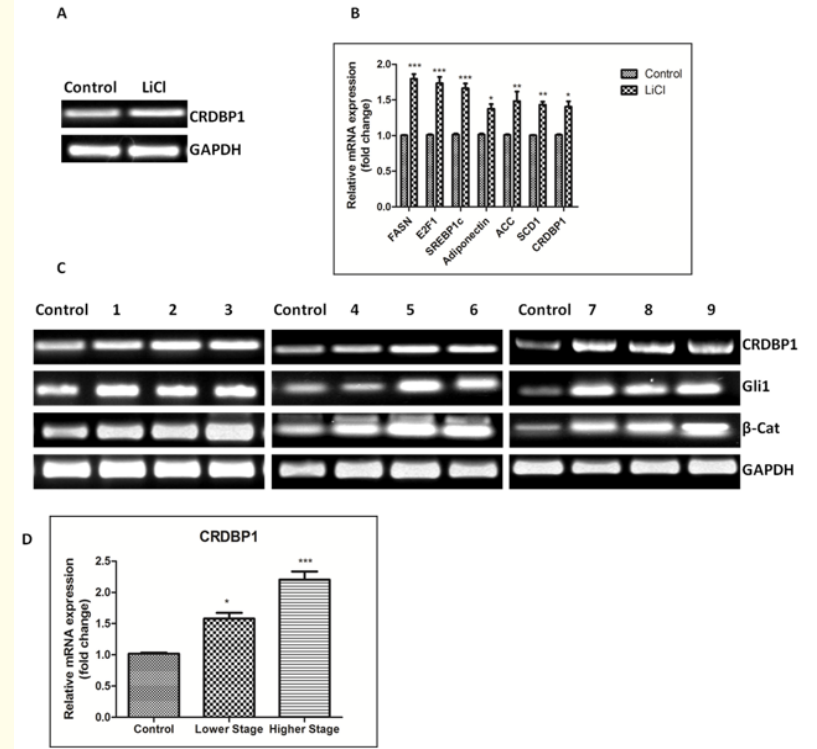

Figure 7: Enhaced Wnt/ $\beta$-catenin activation correlates with increased CRD-BP and Gli1 in HCC patients' biospecimen. (A-B) The mRNA expression level of $\beta$-catenin, CRD-BP and Gli1 after LiCl treatment in Hep3B cells. (C-D) The mRNA expression level of $\beta$-catenin, CRD-BP and Gli1 in FNAC patients samples of different HCC stages respectively. Data represented are representative of three independent experiments performed in triplicates and expressed as Mean $\pm S E,{ }^{*} \mathrm{p}<0.05,{ }^{* *} \mathrm{p}<0.001,{ }^{* * *} \mathrm{p}<0.0001$.

Wnt inhibition resulted into reduced Hh signalling and consequent tumorigenesis in DEN induced rodent hepatocarcinogenesis model

We further investigated the impact of Wnt $/ \beta$-catenin pathway inhibition in DEN induced rodent hepatocarcinogenesis model. The animals were treated with DEN+CCl4 in presence and absence of SS. The results revealed decrease in the number and size of nodules in SS treated group (Figure 8A) and this decrease was associated with significantly reduced expression of Wnt and Hh pathway molecules at protein (Figure 8B-C) and mRNA level (Figure 8D-E).

\section{Discussion and Conclusion}

A comprehensive understanding of interaction between Wnt and Hh pathways in HCC is important to target the best molecular therapeutic spectrum for a relapse-free HCC treatment. Based on the relevant literatures regarding the functional interaction between Wnt and Hh pathways in various cancer models, the interaction between these two pathways could be either positive or negative [25]. In few cancer models Wht is upstream of Hh [10] and in other cancers $\mathrm{Hh}$ is upstream of Wnt [26]. Additionally, in an in vitro study with gastrointestinal and lung cancer cells the activation of Wnt signalling pathway has been found to be associated with enhanced expression and activity of Hh signalling pathway [27]. Furthermore, Teissedre., et al. (2009) demonstrated that activation of Wnt pathway correlates with the stimulation of $\mathrm{Hh}$ pathway and this interaction play important role in tumorigenesis

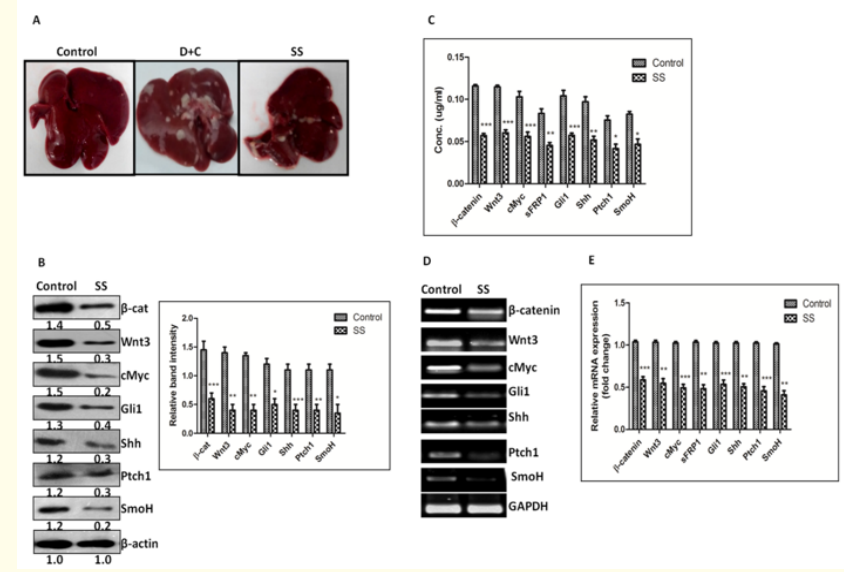

Figure 8: Wnt inhibition resulted into reduced Hh signaling and consequent tumorigenesis in DEN induced rodent hepatocarcinogenesis model. (A) Formation of hepatic foci/nodules in DEN+ CCL4 and SS treated rats. (B) Western blot analysis of Wnt-Hh molecules in SS treated groups. The graph showing relative band intensity. (C) Quantification of Wnt-Hh molecules in SS treated rat tissue lysates by ELISA. (D-E) Fold decrease in relative mRNA expression, calculated with respect to GAPDH for wnt-Hh molecules in SS treated groups. Data represented are representative of three independent experiments performed in triplicates and expressed as Mean $\pm \mathrm{SE},{ }^{*} \mathrm{p}<0.05,{ }^{* *} \mathrm{p}<0.001,{ }^{* * *} \mathrm{p}<0.0001$.

in breast cancer mice model [28]. All these studies indicate that the nature of Wnt-Hh interaction is complex, cell-context, and cancertype dependent.

In our study we identified the functional relation between WntHh pathways in HCC cell-line and substantiated this finding with animal model of hepatocarcinogenesis. Activation of Wnt signalling pathway appears to be a key driver for development of HCC [6]. Our group and others have demonstrated the activation of $\mathrm{Wnt} / \beta$ catenin pathway plays an important role in initiation of HCC in rodent model [6]. Activation of Hh pathway is also observed in various cases of HCC including rodent model; however, the activation of Hh pathway occurs little later than Wnt pathway in rodent hepatocarcinogenesis model [6]. In agreement with our previous study we found a positive correlation between Wnt and Hh pathways where Wnt worked upstream of Hh pathway. Our results demonstrated inhibition of Wnt/ $\beta$-catenin pathway with SS resulted in decreased short-term and long-term cell survival (Figure 1A-B) along with decrease in the expression of downstream target genes. Similarly, the inhibition of Hh pathway with $\mathrm{KC}$ resulted in decreased shortterm and long-term cell survival (Figure 2A-B) and simultaneous decrease in the expression downstream target genes. Interestingly, the effect of co-treatment with Wnt and Hh pathway inhibitors was synergistic on Hep3B cells with a pronounced decrease in percentage cell viability and number of colonies in comparison to either one inhibitor treatment (Figure 3A-B). 
Furthermore, to elaborate the role of Wnt signalling pathway in pathogenesis of HCC and its interaction with Hh pathway we performed in-vitro and in-vivo experiments. First, we exogenously activated Wnt pathway by using $\mathrm{LiCl}$ in Hep3B cell line. LiCl treatment led to Wnt signalling pathway activation and enhanced accumulation $\beta$-catenin in nucleo-cytoplasmic region of cells. This was further associated with up-regulation of Wnt target genes (Figure $4 \mathrm{~A}$ ). Interestingly, the up-regulation of Wnt signalling pathway was positively correlated with the up-regulation of Hh signalling pathway, as the direct transcriptional target of Hh-pathway (Gli1 and Ptch1) was also increased (Figure 4B-F). This result indicates positive correlation between Wnt-Hh signalling pathways in Hep3B cells. Moreover, we also inhibited $\beta$-catenin in Hep3B cells through siRNA which resulted in reduced Wnt and Hh target genes (Figure 5,6$)$. Notably, this decrease in $\beta$-catenin was partially reversed when cells were treated with LiCl (Figure 5,6).

CRD-BP (Coding Region Determinant Binding Protein) is an oncofetal protein and is a direct transcriptional target of Wnt signalling pathway. Our results demonstrated that CRD-BP is overexpressed when Hep3B cells were treated with $\mathrm{LiCl}$ and this increase was further positively correlated with increased expression of Gli1. The simultaneous increase in $\beta$-catenin, CRD-BP and Gli1 implicate the role of CRD-BP as a connecting molecule between Wnt-Hh signalling pathways and imparts a positive correlation between these two pathways in Hep3B cells. Noubissi., et al. (2014) demonstrated that $\mathrm{Wnt} / \beta$-catenin signalling pathway up-regulates CRD-BP, which in-turn bind to and stabilizes Gli1 mRNA. Thus Wnt pathway consequently upregulates expression and activity of Gli1, a Hh target gene [29]. We further checked the expression level of CRD-BP in HCC patients' samples. FNAC samples from HCC patients and control patients were analyzed for the expression level of $\beta$-catenin, CRD-BP and Gli1. The results revealed higher expression level of all three molecules in all HCC patients compared to control (Figure 7A-D). In this way our result provided a proof of concept for the role of CRD-BP in hepatocarcinogenesis.

$\mathrm{DEN}+\mathrm{CCl} 4$ induced rodent hepatocarcinogenesis model is one of the well characterized model to study the molecular changes associated with hepatocarcinogenesis and effect of inhibitors on the same. We established DEN+CCl4 induced hepatocarcinogenesis model in our lab and studied the chemo-preventive effect of SS on HCC of male wistar rats. Our results demonstrated SS treatment significantly reduced the size and number of nodules in the liver (Figure 8A). Besides, the expression level of Wnt signaling pathway molecules was also significantly reduced. More interestingly, the expression levels of Hh signalling pathway molecules were also reduced simultaneously (Figure 8B-E). Few studies have been done in animal model of colorectal cancers to elucidate the molecular mechanism of chemo-preventive action of SS [30]. However, up till now none of the studies have shown the efficacy of SS in preventing HCC in rodent model. We have explored the potential utility of
SS as a chemo-preventive agent in HCC by targeting Wnt signalling pathway. Our results support the notion that SS inhibits rodent hepatocarcinogenesis by reducing Wnt signalling pathway and consequently Hh signalling pathway.

Multiple signalling pathways interact/cross talk with each other in cancer to provide a facile and productive ground for cancer initiation and progression. Such studies to elucidate the interaction between signalling pathways provides a key for development of better therapeutics. Our study elaborated the nature of cross-talk between two well known aberrant signalling pathways in HCC which would further pave the way for development of better combination therapy approach for targeting these two pathways in HCC.

\section{Author Contributions}

AT performed all the experiments. MKS, KU, AS, and JN were involved in providing patients' sample and participated in patients' data analysis. RK conceived the study, participated in its design and coordination, supervised the project and wrote the first draft of the manuscript. All the authors were involved in drafting the manuscripts and its critical revision. All the authors read and approved the final manuscript for publication.

\section{Acknowledgements}

We thank Department of Biotechnology, Government of India (http://www.dbtindia.nic.in/) for providing financial support to carry out this work.

\section{Conflicts of Interest}

The authors declare that they have no competing interest.

\section{Funding}

Grant received BT/Bio-CARe/07/567/2011-12 from Department of Biotechnology, Government of India (http://www.dbtindia.nic.in/).

\section{Bibliography}

1. Kumari R., et al. "Hepatocellular carcinoma treatment: hurdles, advances and prospects". Hepatology Oncology 5.2 (2018): HEP08.

2. Kumari R., et al. "Mitomycin C induces bystander killing in homogeneous and heterogeneous hepatoma cellular models". Molecular Cancer 8 (2009): 87.

3. Jiapei Guo., et al. "Mechanisms of resistance to chemotherapy and radiotherapy in hepatocellular carcinoma". Translational Cancer Research 7.3 (2018): 765-781.

4. Ghouri YA., et al. "Review of hepatocellular carcinoma: Epidemiology, etiology, and carcinogenesis". Journal of Carcinogenesis 16 (2017): 1.

5. M Setshedi., et al. "Molecular and cellular oncogenic mechanisms in hepatocellular carcinoma'. South African Medical Journal 108 (2018): S41-S46. 
6. Tripathy A., et al. "The molecular connection of histopathological heterogeneity in hepatocellular carcinoma: A role of Wnt and Hedgehog signaling pathways". PLoS One 13.12 (2018): e0208194.

7. Ingham PW and McMahon AP. "Hedgehog signaling in animal development: paradigms and principles". Genes and Development 15 (2001): 3059-3087.

8. Pasca di Magliano M., et al. "Common activation of canonical Wnt signaling in pancreatic adenocarcinoma". PLoS One 2.11 (2007): e1155.

9. Song L., et al. "Crosstalk between Wnt/ $\beta$-catenin and Hedgehog/Gli signaling pathways in colon cancer and implications for therapy". Cancer Biology Therapy 16.1 (2015): 1-7.

10. Noubissi FK., et al. "Cross-Talk between Wnt and Hh Signaling Pathways in the Pathology of Basal Cell Carcinoma". International Journal of Environmental Research and Public Health 15.7 (2018): 1442.

11. Giakoustidis A., et al. "Molecular signalling in hepatocellular carcinoma: Role of and crosstalk among WNT/ß-catenin, Sonic Hedgehog, Notch and Dickkopf-1". Canadian Journal of Gastroenterology and Hepatology 29.4 (2015): 209-217.

12. Goldsberry WN., et al. "A Review of the Role of Wnt in Cancer Immunomodulation". Cancers (Basel) 11.6 (2019): 771.

13. Della Corte CM., et al. "Implication of the Hedgehog pathway in hepatocellular carcinoma". World Journal of Gastroenterology 23.24 (2017): 4330-4340.

14. Li X., et al. "Sulindac sulfide inhibits colon cancer cell growth and downregulates specificity protein transcription factors". BMC Cancer 15 (2015): 974.

15. Jason D., et al. "A Novel Sulindac Derivative that Potently Suppresses Colon Tumor Cell Growth by Inhibiting cGMP Phosphodiesterase and $\beta$-Catenin Transcriptional Activity". Cancer Preventive Research (2012): 822-833.

16. Lee HJ., et al. "Sulindac inhibits canonical Wnt signaling by blocking the PDZ domain of the protein Dishevelled". Angewandte Chemie International Edition 48.35 (2009): 64486452.

17. Chen JK., et al. "Inhibition of Hedgehog signaling by direct binding of cyclopamine to Smoothened". Genes and Development 16.21 (2002): 2743-2748.

18. Sari IN., et al. "Hedgehog Signaling in Cancer: A Prospective Therapeutic Target for Eradicating Cancer Stem Cells". Cells 7.11 (2018): 208.

19. Xie H., et al. "Recent Advances in the Clinical Targeting of Hedgehog/GLI Signaling in Cancer". Cells 8.5 (2019): 394.
20. Stambolic V., et al. "Lithium inhibits glycogen synthase kinase-3 activity and mimics wingless signalling in intact cells". Current Biology 6 (1996): 1664-1669.

21. O'Brien WT and Klein PS. "Validating GSK3 as an in vivo target of lithium action". Biochemical Society Transactions 37 (2009): 1133-1138.

22. Derksen PW., et al. "Illegitimate WNT signaling promotes proliferation of multiple myeloma cells". Proceedings of the National Academy of Sciences of the United States of America 101.16 (2004): 6122-6127.

23. Loebel A., et al. "Lurasidone as adjunctive therapy with lithium or valproate for the treatment of bipolar i depression: A randomized, double-blind, placebo-controlled study". American Journal of Psychiatry 171 (2014): 169-177.

24. Huang X., et al. "Insulin-like growth factor 2 mRNA-binding protein 1 (IGF2BP1) in cancer". Journal of Hematology and Oncology 11.1 (2018): 88.

25. Thompson M., et al. "Crosstalk of the Wnt Signaling Pathway". In: Goss K., Kahn M. (eds) Targeting the Wnt Pathway in Cancer. Springer, New York, NY (2011).

26. Liao X., et al. "Aberrant activation of hedgehog signaling pathway contributes to endometrial carcinogenesis through $\beta$-catenin". Modern Pathology 22 (2009): 839-847.

27. Maeda 0., et al. "Enhancement of GLI1-transcriptional activity by $\beta$-catenin in human cancer cells'. Oncology Reports 16 (2006): 91-96.

28. Teissedre B., et al. "MMTV-Wnt1 and $-\Delta \mathrm{N} 89 \beta$-Catenin Induce Canonical Signaling in Distinct Progenitors and Differentially Activate Hedgehog Signaling within Mammary Tumors". PLoS One 4.3 (2009): 10.

29. Noubissi FK., et al. "Role of CRD-BP in the growth of human basal cell carcinoma cells". Journal of Investigative Dermatology 134.6 (2014): 1718-1724.

30. Williams CS., et al. "Sulindac Sulfide, but not Sulindac Sulfone, Inhibits Colorectal Cancer Growth". Neoplasia 1.2 (1999): 170176.

\section{Assets from publication with us}

- Prompt Acknowledgement after receiving the article

- Thorough Double blinded peer review

- Rapid Publication

- Issue of Publication Certificate

- High visibility of your Published work

Website: www.actascientific.com

Submit Article: www.actascientific.com/submission.php Email us: editor@actascientific.com Contact us: +91 9182824667 